\title{
The Research on Hidden Failures of Civil Aircraft
}

\author{
Lie Shu LIN, Shuang Xue FU ${ }^{\text {a }}$ \\ Guangzhou Civil Aviation College, Guangzhou, Guangdong, 510403, China
}

\begin{abstract}
The hidden failure is often appear on civil aircraft, and it is a big risk for normal safe operation. The reason for this failure is the instability of the resistance of the electrical contact. The study on electrical contact is applied science of the reliability, the contact resistance is an important parameter of the reliability of the electrical contact system. In this paper, It also Produces the latest research results from factors that affect contact resistance and contact resistance microscopic model, and then it produces the method of the calculating and measuring engineering about the contact resistance. Lastly, it is point out the future direction of the electrical contacts.
\end{abstract}

Key words: Aircraft maintenance; Electrical contact; Contact resistance; Reliability.

\section{INTRODUCTION}

Hidden failures in the aircraft airborne equipment occur, are as the following common scenarios: (1) The operation of the airborne equipment in the air is not normal, while check everything is normal on the ground when power is on; (2) Equipment' operation is well when the power is on , and some time is over, failure occur suddenly, and then disappear; (3) the equipment occasionally did not work properly in the air;(4) In several successive days of flight, the equipment is working properly, and in a certain program instantaneous flight irregularities occur; (5) The equipment in a particular state is not normal randomness. Factors produced such failures are roughly divided into three categories:(a). Failure of the equipment itself;(b). improper influence of external conditions; (c) and / or using. Among them, the failure of the equipment itself is the main reason causing hidden failure of the aircraft, and its essence is the point of electrical connection in equipment, the characteristics of the contact resistance value is unstable.

Electrical connections are essential to aircraft systems and there are a lot of links, and electrical contact is the key of electrical connection [1]. Electrical connection can be divided into strong and weak categories .The main function of strong category is the transmission of energy, and electric arc and thermal effect are the main factors affecting the life and reliability of this connection; The main function of weak category is signal transmission, the environment is one of the main factors to the reliability and failure of this electrical connections[2]. Quality and level electrical connector had an important impact on the reliability of the equipment and systems. The electrical contact belong to multi- science. it use physical, mechanical, electrical materials, chemical,

\footnotetext{
a Corresponding author:25272505@qq.com
}

environmental concepts and theories in reliability issues of electrical , find electrical contact theories. Electrical contact is a common phenomenon in which aircraft systems exist .Between the elements of these systems, even between systems of the whole aircraft require electrical connections. If the contact point is in failure, it would affect the entire system reliable operation. This leads to issue about reliability, stability, lifetime of the associated electrical contact. While the contact resistance is a basic and important parameter of electrically connection. This parameter is closely related with the electrical contact material parameter, structure, manufacturing processes, the working environment .Thus, reliability issues caused by the contact resistance of the electrical connection is getting attention by researchers, and achieved some research results. This paper analyzes the affect of an unstable electrical contact resistance, and introduces its micro models for calculating. It has some significance about some guidance to trouble shoot/isolation of Failure in which aircraft [3].

\section{THE CONTACT RESISTOR'S MAJOR FACTORS OF INSTABILITY}

It is important to get stable contact resistance in applications, in order to ensure the reliability of the electrical contact. Factors Affecting the contact resistance value, and the main properties of the conductor material are as following: in the form of the contact, the contact pressure, temperature, corrosion, frequency, and etc.

\subsection{Properties of Materials}

The field for Metal material about electrically contacting is very wide. They are classified by the use, as copper, 
aluminum, and copper-aluminum alloy. They are used in a large current (ferroelectric) contacts in the field, while precious metals and their alloys are mostly used in small current (weak) contact application. Pure precious metal is mainly used as a coating material. Metallic material properties of constituting the electrical contact directly affect the volume of the contact resistance. these properties are the mainly resistivity of the material $\rho$, Material hardness H, etc. Shrink resistance volume of each conductive spot is proportional to $\rho$. The spots number on contact surface of the contact material are decided by material hardness . According to the definition of the hardness $\mathrm{H}$ of the contact material, Relationship between the small contact area of contact surface with the contact pressure $\mathrm{F}$ is as following :

$$
F=\xi H \pi a^{2}
$$

Wherein, $\xi$ is a constant, and less than $1 ; a$ is an radius of the small contact surface.

\subsection{Contact Form}

An electrical contact form can be divided into three categories: point contact, line contact and surface contact. The number of contact points mainly affect a shrink resistor $R_{s}$. In general, the contact points ( $\mathrm{n}$ ) on the contact surface are at Maximum, the shrinkage resistance ( $\left.R_{s}=\rho / 2 n a\right)$ will be minimum, in contrast, the contact points $(\mathrm{n})$ on the contact surface are at minimum, the shrinkage resistance $R_{s}$ will be maximum. The shrinkage resistance $R_{s}$ volume on the line contact is in between the above two volume. The film resistors' volume is mainly affected by contact pressure at each point of the contact surface. If the pressure $\mathrm{F}$ is applied on the contacts, the number of contact points is $n$, then the pressure at each point of contact on, $F_{1}=F / n$, thus, that each pressure on the point of contact is related with the contact form . In general, when the number of the contact points $(n)$ of point contact is at minimum, and under the same contact pressure $\mathrm{F}$ is applied, so each contact point in point contact is in maximum pressure. this is the most likely to damage the contact surface of the contact film, so that the resistance of film is reduced. In contrast, the number of the contact points ( $\mathrm{n}$ ) in surface contact is the maximum , the ability to damage the surface film is small, the film resistance increases. The resistance volume on the line contact is between the point contact and surface contact. Moreover, the contact resistance is also related to the contact pressure. when the contact pressure is in small, the contact resistance volume of the contact surface is not sure smaller than a point or line contact.The data in Table 1 illustrates this point.

Table1. The contact resistance of the contact form of relationship -(copper) [4]

\begin{tabular}{|c|c|}
\hline $\begin{array}{c}\text { Contact } \\
\text { form }\end{array}$ & $R_{j}(u \Omega)$ \\
\hline
\end{tabular}

\begin{tabular}{|c|c|c|}
\hline & $F=9.807(N)$ & $F=980.7(N)$ \\
\hline $\begin{array}{c}\text { Point } \\
\text { contact }\end{array}$ & 230 & $23 R_{j}$ \\
\hline $\begin{array}{c}\text { Line } \\
\text { contact }\end{array}$ & 330 & 15 \\
\hline $\begin{array}{c}\text { Surface } \\
\text { contact }\end{array}$ & 1900 & 1 \\
\hline
\end{tabular}

The vast majority of the pins and jacks installed on the aircraft are made of gold-plated for conduction and protection, their coating is worn after they are frequently removed and installed, the contact resistance $\left(R_{j}\right)$ will been changing. These will Cause circuits' failure. Aircraft manufacturers such as Boeing report:

"SUNDSTRAND company show that: EICAS indicator frequently show "IDG TEMP" phenomenon on Beoing 757,767 display, they are proved that IDG nailing on the joint changed, and led to resulting coating worn [5] .

\subsection{Contact pressure}

We could see from Table 1, the contact pressure $\mathrm{F}$ has an important effect on the contact resistance. If the pressure is not enough, the resistance do not make a Significantly reduce only by increasing the contact surface dimensions When the contact pressure F increased enough, material's compression would be beyond the elastic deformation limits, and produced plastic deformation, then the contact surface would increase, the number of contact point would also increase, $\left(R_{s}=\rho / 2 n a\right)$ would reduced. At the same time, the contact pressure increased can crush the metal film surface, lead to the film resistance reduce. Therefore, increasing the contact pressure, the contact resistance can be reduce. Some parts installed in the joints are subjected to vibration, such as a aircraft wing or an engine joint section, they are frequently subject to vibration for a long time, resulting in looseness between the receptacles, the contact pressure changes, the contact resistance $\left(R_{j}\right)$ will change. Therefore, when the current through the joints, They can produce a heat or even an arc, lead to wholly or partly pins burn, contact resistance increased further. For airborne precision instruments, their indication would result in a blank or swinging.

\subsection{Temperature}

As the contact point temperature raise, the resistivity of the metal will increase, while the hardness of the material will be reduce, so that the effective contact area will increase, and the number of the contact points will increase. The former made volume of resistance increase, the latter reduce. Both mutual compensation, the contact resistance variation is minima. However, if the current at the contact are more than the short-circuit current, the temperature would raise sharply, the contact surface would oxidize, the growth and thickening of the oxide film, the film resistance would increase, produce more heat and More serious consequences. In order to maintain a stable contact resistance, electrical contact with 
predetermined temperature is in very low for long time. Joints installed on aircraft in external environment (such as joints on the engine electrical components), due to the drastic changes in temperature, joints in the middle of the rubber pad are deformed, although the joint external connection is good, but no virtually and fully contact between the jacks or loosen, the system do not operate and display properly. Such as:CSD oil temperature indicator indicated a larger swing in China Southern Airlines B-2926 aircraft recently, the reason is that the joint is un-completely contact or loosed in the middle of the rubber pad by sudden temperature changing lead to aging and deformation[6] .

\subsection{Corrosion}

The external environment around electrical contact also affects the electrical contact. Where in the contact corrosion can seriously affect the electrical contact resistance, and the reliability of the electrical contact. There are three types of the electrical contact corrosion or contamination : pore corrosion, creep corrosion and tarnishing. These three types affect the reliability of electrical contact. Pore Corrosion is a chemical or electrochemical reaction between air pollutants and base metal, it occurres through pores and other weaknesses of the coating surface. Insulating film and the corrosion products formed by the reaction can lead to unstable contact, accelerate friction, the contact resistance increase and so, eventually lead to the electrical contact failure. Copper such as other base metals and precious metals such as gold plating creep corrosion will react after bonding. The characteristics of the reaction process are obviously distribution matrix material in the surface of the gold plating creep corrosion products. Some trends in the metal sulfide generation exceed the oxides trend, the gaseous sulfur-containing substances around coating occurred chemical reaction on the surface of these metals, and produce diffusion phenomena, resulting in metal tarnish. The tarnish will affect electrical contact reliability.

Abbott, Antler and other researchers had extensively studied tarnish phenomenon about effect electrical connectors.

\subsection{Signal frequency[7]}

When the alternating current gone through the contact point, the current only gone through a conductor surface, due to the skin effect . it is more obviously at high frequencies. Skin depth $\delta$ could be calculated as:

$$
\delta=\sqrt{\frac{\rho}{\pi f \mu_{0}}}
$$

Wherein, $\rho$ is the conductor resistivity; $f$ is the frequency of the AC singnal; $\mu_{0}$ is vacuum magnetic conductivity

Figure 1 showed the top contact between two spherical of the same configuration with conductor having the same radius of cylindrical.

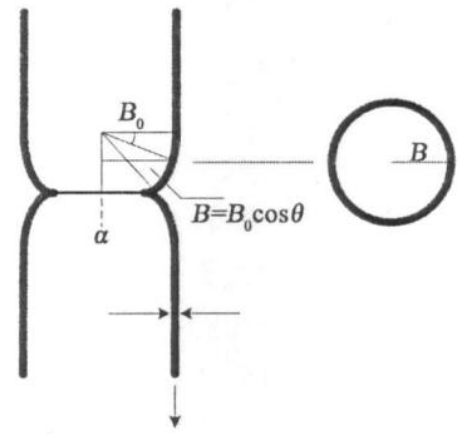

Figure 1. Skin Effect and contraction resistance of the substrate

Assuming skin effect, the effective current only gone through the thin shell in the defined depth of penetration, and assuming the thin shell's size smaller than the radius of the contact pattern, Marucci gave the formula for calculating the extra resistance due to the skin effect.

Wherein, $\rho$ is the conductor resistivity; $\delta$ is the skin depth; $a$ is a minimum radius of two conductors Correspondingly cylinders; $B_{0}$ is the maximum radius with contact cylinder.

\section{ADVANCES CONTACT RESISTANCE MODEL}

\subsection{Holm electrical contact model [8]}

Holm suggested that the total contact resistance should be a parallel value of all actual point of contact resistance (called self-resistance) and the resistance value caused by the interaction (called mutual resistance) in series, namely:

$$
R_{j}=R_{s}+R_{i}=\frac{\rho}{2 \sum_{i=1}^{n} a_{i}}+\frac{\rho}{2 a}=\frac{\rho}{2 n a}+\frac{\rho}{2 a}
$$

In the formula, $R_{j}$ is the total contact resistance, $a$ is Holm radius which called mutual resistance or point set radius. Later, Mr. S .Timoshenko [9] estimated from prosperity by a dense point cloud, $R_{j}=\frac{16 \rho}{3 \pi^{2} a}$, while $\frac{16 \rho}{3 \pi^{2}}=0.5404 \approx \frac{1}{2}$. The result is consistent with Holm equation.

\subsection{Green Wood electrical contact model [10]}

Green Wood gave the formula for calculating the contact resistance if that the current gone through the contact point between the circle model :

$$
R_{G 1}=\frac{\rho}{2 \sum a_{i}}+\frac{\rho}{\pi} \sum_{i \neq j} \sum \frac{a_{i} a_{j}}{d_{i j}} / \sum a_{j}^{2}=R_{p a r}+R_{\mathrm{int}}
$$

Where, $\rho$ is the resistivity, $a_{i}$ is the $\mathrm{i}$-th point of the radius, $d_{i j}$ is distance between the $\mathrm{i}$ - point and the $\mathrm{j}$-th 
point of circles, $R_{\text {par }}$ is the parallel resistance of the dependent point of circle, and $R_{\text {int }}$ is resistance of the each point between the interaction circles, If it is assumed that all circles are the same size, then the above equation can be approximated as:

$$
R_{G 2}=\frac{\rho}{2 \sum a_{i}}+\frac{\rho}{\pi \cdot n} \sum_{i \neq j} \sum \frac{1}{d_{i j}}
$$

In 2001, Lionel Boyer [11] Promoted the use of the formula given by Greenwood through adding a shape factor $\mathrm{S}(\mathrm{N})$, such that the formula given by Greenwood could also be used to calculate the contact resistance to other conductive spots, Wherein the square form factor, $S(4)=\left(2+\frac{1}{\sqrt{2}}\right) / \pi=0.8617$, is very close to the 0.868 about Nakamura's. While, the triangular shape factor, $T(4)=3(1+\sqrt{3}) / 2 \pi=1.3$, is close $R_{T N}\left(R_{T N}=1.27 \rho / L\right)$ in the reference, Nakamura[12].

\section{3 .3 The Electrical contact for surface film}

The electrical contact surface is covered with a poorly conductive material due to various factors, such as metal oxides, sulfides, dust, dirt, or sandwiched between the contact surfaces of the oil film, water film, etc. In general, the more oxide film cover with electrical contact surface. This oxide film is mostly semiconductor, and its resistivity is high. Because of the conductive film layer on the contact surface, while shrink resistance is advantages or disadvantages, depending on the resistivity between the film and the substrate The film can also change the hardness of the contact surface, and the deformation of the contact surface can change the contact resistance when the contact surface of film is thiner enough, and the film strength of the electric field will less than $10^{8} \sim 10^{9} \mathrm{v} / \mathrm{m}$, the resistivity of the tune of film from the Schrodinger equation:

$$
\sigma=\frac{U}{J}
$$

Wherein, $\mathrm{U}$ is the voltage between the contact surface; $\mathrm{J}$ is the current density flowing through the membrane. The electrons pass through the membrane resistance spot radius of a surface is encountered:

$$
R_{b}=\frac{U}{I}=\frac{U}{J \pi a^{2}}=\frac{\sigma}{\pi a^{2}}
$$

If there are $n$ conductive spots, the mean radius of the inner contact surface is $a_{p}$, then the total resistance of the film is as following:

$$
R_{b}=\frac{\sigma}{\pi a^{2} a_{p}^{2}}
$$

\section{CONTACT RESISTANCE CALCULATING}

Contact resistance consisted of two parts. The value of the total resistance is shrinkage resistance $\left(R_{S}\right)$ plus resistance of the surface film ( $R_{b}: R_{j}=R_{s}+R_{b}$ ). However, due to ( $\mathrm{n}$ ) the number of spots and the average radius are very difficult, so In general to calculate the contact resistance for engineering is as the following empirical formula:

$$
R_{j}=\frac{k_{j}}{0.192 F^{m}}
$$

Where, $R_{j}$ is contact resistance; $F$ is the contact pressure; $m$ is the actual index relating to the contact form, pressure and number of points of contact (proved, if the pressure is not too large, for point contact with $m$ $=0.5$; line contact with $m=0.5 \sim 0.8$ (generally the $m=$ 0.7 ); surface contact with $m=1 ; k_{j}$ is the relevant factor for the contact material, surface condition, etc. Whose value is determined by the experiment. It is shown in Table 2.

Table 2. Values of $k_{j}$ for various contact materials

\begin{tabular}{|c|c|c|}
\hline Contact materials & $\begin{array}{c}\text { Surface } \\
\text { condition }\end{array}$ & $k_{j}$ \\
\hline$A_{g}-A_{g}$ & unoxidized & 0.06 \\
\hline$A l-C u$ & unoxidized & 0.98 \\
\hline$C u-C u$ & unoxidized & $0.08 \sim 0.14$ \\
\hline$A l-b r a s s$ & unoxidized & 1.90 \\
\hline$A_{g} C_{d} O(12)-A_{g} C_{d} O(12)$ & unoxidized & 0.17 \\
\hline$A_{g} C_{d} O(12)-A_{g} C_{d} O(12)$ & oxidized & 0.35 \\
\hline
\end{tabular}

It must be note, there is significant limitations of this formula, we can not generalize the influence of various factors on the contact resistance. Contact resistance are frequently measured by measuring the contact voltage in application.

Figure 2 show a circuit diagram for measuring contact resistance. The $R_{j}$ is contact resistance of electrical connector, $V_{m}$ is electrical contact voltage measured, $V_{\text {in }}$ is the supply voltage, the contact resistance can be calculated as:

$$
R_{j}=\frac{V_{m} R}{V_{\text {in }}-V_{m}}
$$

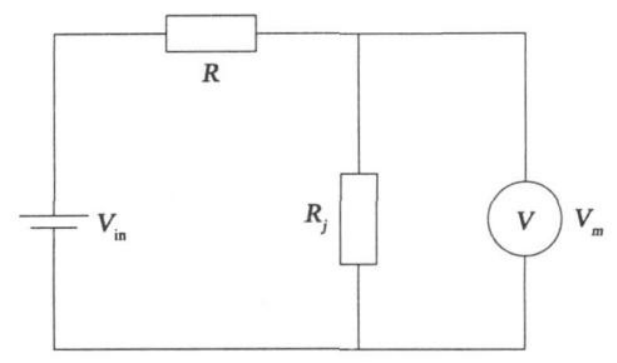

Figure 2. Showed a circuit diagram for measuring contact resistance

\section{SUMMARY}

There are a lots of high frequency signals used in the various aircraft systems, particularly in electronic 
systems. The study is not sufficient about the impact of the high frequency to the contact resistance, particularly in the case of multiple points of contact. As frequency increased, the contact resistance with only a parameter which is difficult to describe the good electrical contact of the model, then you need to study the electrical impedance model of the contact, including contact with capacitors and inductors. Although the research in this area has been reported, but it still need further studying.

Further, the contact nanoscale with the rapid development of nano-size electronic devices, especially with the microelectro mechanical system (MEMS) development, research issues related to the electrical contact became more urgent and more valuable.

\section{ACKNOWLEDGMENTS}

The authors gratefully acknowledge the support of training program of Guangdong province outstanding young teachers in colleges and universities (YQ2014178).

\section{References}

1. Airframe and Powerplant Mechanics General Handbook US Department of Transportation. Printed by ASA Publication,INC

2. (Canada) Brown Novick, (Belarus) Kangqi Zi, (Russia) with Mishkin, Xu Liangjun translated electrical contact theory, application and technology Beijing: Mechanical Industry Press(2010).

3. Turbine engine aircraft structures and systems. Ordnance Industry Press, ISBN-7-80172-766-5

4. Guo Fengyi, Zhonghua. Electrical contact theory and its application technology, Beijing: Electric Power Press,(2008).

5. Aviation accidents and human factors . China Civil Aviation Press, ISBN7-80110-443-9 / V

6. Aviation education ISSN 1005-8176 / CN11-3411 / G4 (Total No. 59)

7. Malucci R D .High Frequency Considerations for Multi-Point Contact Interfaces .Proceeding of the FortyFifthIEEE Holm Conference on Electrical Contact s (2001) .

8. Holm R.Electrical Contacts. New York:Springer(1979).

9. Timoshen ko S, Goodier J N .Theory of Elasticity .New York: McGraw Hill (1951).

10. Greenwood J A .Constriction Resistance and the Real Area of Contact .Brit .J .App 1.Phys , 17:1621 1632 (1966).

11. Lionel Boyer .Contact Resistance Calculations: Generalizations of Greenwood's Formula Including Interface Films .I EEE Transact ions on Components and Packing Technologies , 24(1):50-59 (2001).

12. Nakamura M, Minowa I .Computer Simulation for the Conductance of a Contact Interface.IEEE Transactionson Components , Hybrids and Manufacturing Technology, CHM T $\quad$-9:150155(1986) . 